\title{
Incorporating Emotion and Personality-Based Analysis in User-Centered Modelling
}

\author{
Mohamed Mostafa, Tom Crick, Ana C. Calderon, and Giles Oatley
}

\begin{abstract}
Understanding complex user behaviour under various conditions, scenarios and journeys can be fundamental to the improvement of the user-experience for a given system. Predictive models of user reactions, responses - and in particular, emotions - can aid in the design of more intuitive and usable systems. Building on this theme, the preliminary research presented in this paper correlates events and interactions in an online social network against user behaviour, focusing on personality traits. Emotional context and tone is analysed and modelled based on varying types of sentiments that users express in their language using the IBM Watson Developer Cloud tools. The data collected in this study thus provides further evidence towards supporting the hypothesis that analysing and modelling emotions, sentiments and personality traits provides valuable insight into improving the user experience of complex social computer systems.
\end{abstract}

\section{Introduction}

As computer systems and applications have become more widespread and complex, with increasing demands and expectations of ever-more intuitive human-computer interactions, research in modelling, understanding and predicting user behaviour demands has become a priority across a number of domains. In these application domains, it is useful to obtain knowledge about user profiles or models of software applications, including intelligent agents, adaptive systems, intelligent tutoring systems, recommender systems, e-commerce applications and knowledge manage-

Mohamed Mostafa · Tom Crick · Ana C. Calderon

Department of Computing \& Information Systems, Cardiff Metropolitan University, Cardiff, UK; e-mail: \{momostafa,tcrick, acalderon\}@ cardiffmet.ac.uk

Giles Oatley

School of Engineering \& Information Technology, Murdoch University, Australia;

e-mail: g.oatley@murdoch.edu.au 
ment systems [27]. Furthermore, understanding user behaviour during system events leads to a better informed predictive model capability, allowing the construction of more intuitive interfaces and an improved user experience. This work can be applied across a range of socio-technical systems, impacting upon both personal and business computing.

We are particularly interested in the relationship between digital footprint and behaviour and personality $[2,3,14,17]$. A wide range of pervasive and often publicly available datasets encompassing digital footprints, such as social media activity, can be used to infer personality [11, 19]. Big social data offers the potential for new insights into human behaviour and development of robust models capable of describing individuals and societies [6]. Social media has been used in varying computer system approaches; in the past this has mainly been the textual information contained in blogs, status posts and photo comments [2,3], but there is also a wealth of information in the other ways of interacting with online artefacts. Research in image or video analysis includes promising studies on YouTube videos for classification of specific behaviours and indicators of personality traits [1]. This work uses crowdsourced impressions, social attention, and audiovisual behavioural analysis on slices of conversational video blogs extracted from YouTube. From sharing and gathering of information and data, to catering for marketing and business needs; it is now widely used as technical support for computer system platforms.

The work presented in this paper is building upon previous work in psycholinguistic science (the study of the psychological and neurobiological factors that enable humans to acquire, use, comprehend and produce language) and aims to provide further insight into how the words and constructs we use in our daily life and online interactions reflect our personalities and our underlying emotions. As part of this active research field, it is widely accepted that written text reflects more than the words and syntactic constructs, but also conveys emotion and personality traits [24]. As part of our work, the IBM Watson Tone Analyzer (part of the IBM Watson Developer Cloud toolchain) has been used to identify emotion tones in the textual interactions in an online system, building on previous work in this area that shows a strong correlation between the word choice and personality, emotions, attitude and cognitive processes, providing further evidence that it is possible to profile and potentially predict users identity [8]. The Linguistic Inquiry and Word Count (LIWC) psycholinguistics dictionary [25, 28] is used to find psychologically meaningful word categories from word usage in writing; the work presented here provides a modelling and analysis framework, as well as associated toolchain, for further application to larger datasets to support the research goal of improving user-centered modelling.

The paper is structured as follows: in Section 2 we give an overview of how insight into personality can be inferred from textual data; in Section 3 we introduce the tools, namely the IBM Watson Developer Cloud toolchain; in Section 4 we present our data and the statistical analysis; in Section 5 we identify the key elements of our model and in Sections 6 and 7 we highlight the conclusions and main contributions of this paper, as well as clear recommendations for future research. 
Incorporating Emotion and Personality-Based Analysis in User-Centered Modelling

\section{Personality Insight}

Numerous studies have suggested key words and phrases can signal underlying tendencies and that this can form the basis of identifying certain aspects of personality [10, 20, 21, 24]. Scherer [26] introduced a valuable classification with the following distinctions between emotions, moods, interpersonal stances, attitudes and personality traits:

- Emotion: short-lived, for instance being angry, sad, or joyful;

- Mood: longer-lived, low-intensity for instance being cheerful or gloomy;

- Interpersonal stances: duration linked to specific interaction, for instance friendly or supportive;

- Attitudes: long-lived linked to specific people or events, for instance loving and hating;

- Personality traits: stable personality dispositions and typical behaviour tendencies, for instance nervous, anxious, or hostile.

By observing the occurrences of words that relate to these five categories, we can conclude infer aspects of the holder's psychological state. For instance, we have sentiment analysis or opinion mining, utilising open-source software such as SentiWordNet; as well as the use of features from psycholinguistic databases such as LIWC [25] to create a range of statistical models for each of the Five Factor personality traits [12]. This "Big Five" model, focuses on five dimensions, namely: Agreeableness, Conscientiousness, Extraversion, Neuroticism and Openness [13]:

- Agreeableness (friendly/compassionate vs. analytical/detached) is the tendency to be compassionate and cooperative rather than suspicious and antagonistic. It is also a measure of one's trusting and helpful nature; high agreeableness is often seen as naive or submissive, whereas low agreeableness personalities are often competitive or challenging people, which can be seen as argumentative or untrustworthy.

- Conscientiousness (efficient/organized vs. easy-going/careless) is the tendency to be organised and dependable, showing self-discipline, acting dutifully, aiming for achievement, and preferring planned rather than spontaneous behaviour. High conscientiousness is often perceived as stubborn and obsessive, whereas low conscientiousness personalities are flexible and spontaneous, but can be perceived as sloppy and unreliable.

- Extraversion (outgoing/energetic vs. solitary/reserved) is the tendency to seek stimulation in the company of others, and talkativeness; high extraversion is often perceived as attention-seeking and domineering, whereas low extraversion causes a reserved, reflective personality, which can be perceived as aloof or selfabsorbed.

- Neuroticism (sensitive/nervous vs. secure/confident) is the tendency to experience unpleasant emotions easily, such as anger, anxiety, depression, and vulnerability. Neuroticism also refers to the degree of emotional stability and impulse control and is sometimes referred to as "emotional stability". 
- Openness to experience (inventive/curious vs. consistent/cautious) reflects the degree of intellectual curiosity, creativity and a preference for novelty and variety a person has. High openness can be perceived as unpredictability or lack of focus, whereas those with low openness seek to gain fulfillment through perseverance, and are characterized as pragmatic and data-drivensometimes even perceived to be dogmatic and closed-minded. Some disagreement remains about how to interpret and contextualise the openness factor [23].

It should be noted that while researchers have continued to work with the Five Factors model, there are well known limitations $[4,7,22]$ that are often overlooked; however, over the past fifty years the Five Factor model has become a standard in psychology [12], developing a large corpus of work to compare against.

\section{IBM Watson Developer Cloud Tools}

The IBM Watson Developer Cloud ${ }^{1}$ offers a variety of services across language, speech, vision and data insight for developing cognitive applications; each Watson service provides a REST API for interacting with the service. In this paper, we have used two of the language services - Tone Analyzer and Personality Insights - but also includes natural language classifiers, language translation and retrieve and rank, a machine learning information retrieval tool.

\subsection{IBM Watson Tone Analyzer}

The IBM Watson Tone Analyzer ${ }^{2}$ is a cloud-based framework to infer emotions from a given text; its use cases include: personal and business communications, market research, self-branding and automated contact-center agents. It uses linguistic analysis to detect three types of tones from written text: emotions, social tendencies, and writing style. Emotions identified include Anger, Fear, Joy, Sadness and Disgust; identified social tendencies include the Big Five personality traits (as described above); identified writing styles include Confident, Analytical and Tentative.

To derive emotion scores from text, IBM Watson Tone Analyzer uses a stacked generalisation-based ensemble framework to achieve greater predictive accuracy [5]. Features such as n-grams (unigrams, bigrams and trigrams), punctuation, emoticons, curse words, greeting words (such as "hello", "hi" and "thanks") and sentiment polarity are fed into machine learning algorithms to classify emotion categories [9]. The analysis performed by the Tone Analyzer service is different from sentiment and emotion analyses. Sentiment analysis can identify the positive and negative sentiments within a document or web page. The sentiments can include document-level

\footnotetext{
${ }^{1}$ http://www.ibm.com/watson/developercloud/

2 http://www.ibm.com/watson/developercloud/tone-analyzer.html
} 
sentiment, sentiment for a user-specified target, entity-level sentiment, and keywordlevel sentiment. Emotion analysis can infer different categories of emotions such as joy, anger, disgust, sadness, and fear. The Tone Analyzer service computes emotional tones, in addition to social and writing style tones. LIWC is then used to find psychologically meaningful word categories from word usage in writing.

\subsection{IBM Watson Personality Insights}

IBM Watson Personality Insights ${ }^{3}$ provides a deeper understanding of people's personality characteristics, needs, and values to drive personalisation. It enables applications to derive insights from social media, enterprise data, or other digital communications by extracting and analysing a spectrum of personality attributes to help discover actionable insights about people and entities. The service can automatically infer, from potentially noisy social media, portraits of individuals that reflect their personality characteristics.

The service outputs personality characteristics that are divided into three dimensions: the Big Five, Values and Needs. As described in Section 2, the five primary (top-level) dimensions (Agreeableness, Conscientiousness, Extraversion, Emotional Range and Openness) have six subdimensions, or facets, that further characterise an individual according to the dimension. A Personality Insights portrait can only be created where sufficient data of suitable quantity and quality is provided. Because language use varies naturally from document to document, a small sample of text might not be representative of an individual's overall language patterns. Moreover, different characteristics and different media converge at somewhat different rates. While some services are contextually specific depending on the domain model and content, Personality Insights usually only requires a minimum of 3000+ words of any text, but ideally reflective in nature. The percentile and sampling error provide normalised scores that describe the extent to which the author's writing exhibits a characteristic and the possible range of deviation. They indicate an interval in which the service has $95 \%$ confidence in its results; the raw score and raw sampling error provide similar results.

\section{Data Analysis \& Feature Extraction}

\subsection{Overview of the Data}

Our dataset comes from an online portal for a European Union international scholarship mobility hosted at a UK university. The dataset was generated from interactions

\footnotetext{
${ }^{3} \mathrm{http} / / /$ www.ibm.com/watson/developercloud/personality-insights.html
} 
between users and a complex online information system, namely the online portal for submitting applications.

The whole dataset consists of users $(n=391)$, interactions and comments $(n=1390)$ as responses to system status and reporting their experience with using the system. Google Analytics has been used to track user behaviour and web statistics (such as impressions); this data from has been used to identify the server's status and categorised the status as two stages: Idle, where the system had a higher number of active sessions; and marked as Failure, where the system had a lower number of sessions engaged. Figure 1 provides a plot of web traffic from Google Analytics over a specific day, clearly showing the drop at 20:00 where the system had been identified as in the Failure state.

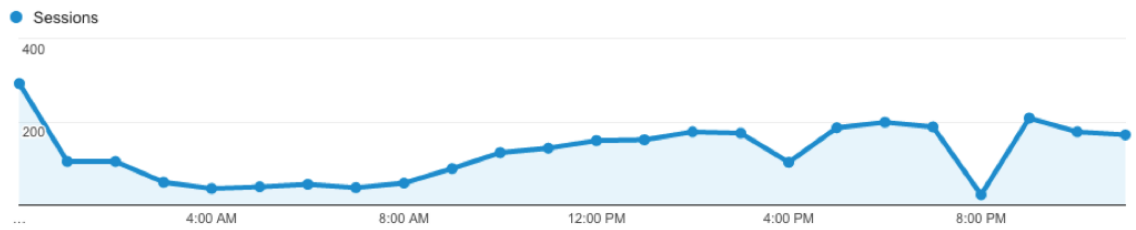

Fig. 1 Google Analytics profile shows behaviour of the system over a 24 hour period (timeline during the day vs. number of active sessions)

All interactions had been collected and grouped by server status, then sent to the IBM Watson Tone Analyzer to generate the emotion social tone scores, to provide an overview of the system behaviour and users interaction with Facebook at the same time. Figure 2 shows the relationship between the server behaviour and emotions of the users; in the system, Failure status shows a significant difference in overall Anger in different status; furthermore, the Joy parameter shows a significant difference with the system in Idle and Failure status. However Fear and Sadness parameters is almost the same, even with the system in Idle status.

We identified the user's personality based on analysis of their Facebook interactions, namely by collecting all comments from the users, again using the IBM Watson Personality Insights tool. However, a number of users in the dataset had completed the Big Five questionnaire $(n=44)$; for these users, their Big Five scores have been used instead. The second stage involved grouping the comments based on server status and segmenting these interactions by user; this allowed us to investigate the impact of server status in the emotion of the user and investigate the Big Five dimension as a constant parameter. By investigating the relationship between personality trait dimensions and the social emotion tones, we are able to find the highest correlation to identify the key elements of the potential model by applying linear regression and Pearson correlation. This will allow building of a neural network multilayer perception using the potential key elements with higher correlations.

The previous overview encourages further investigation to understand the relationship between user's behaviour and complex computer system behaviours. The 
Incorporating Emotion and Personality-Based Analysis in User-Centered Modelling

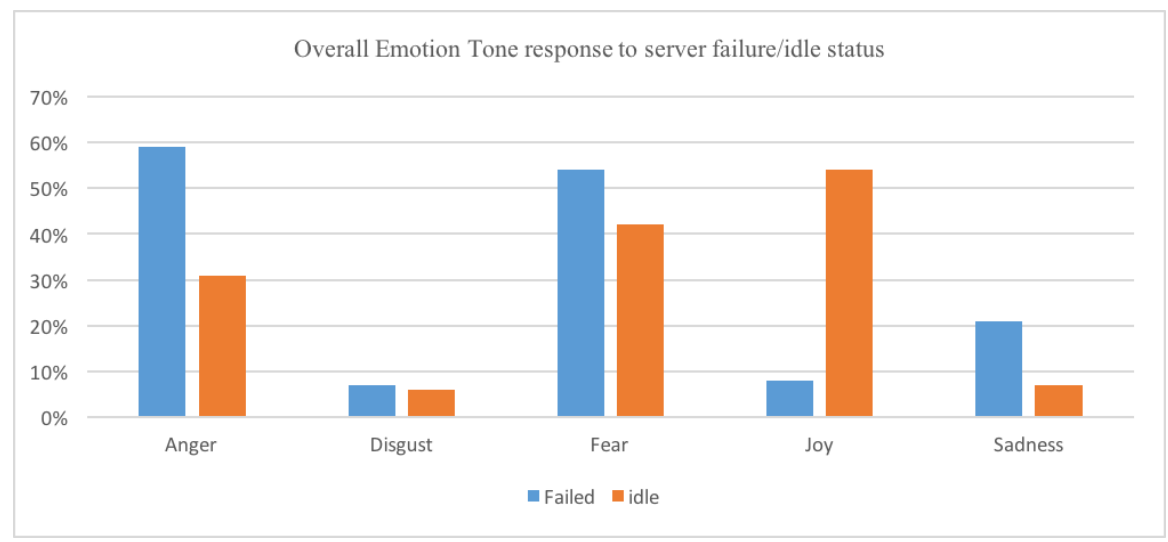

Fig. 2 Overall emotion tone response to server failure/idle status

data collected from the social media interactions have been grouped by users and using the IBM Watson Personality Insights, we were able to identify the Big Five personality traits for each user. Using the IBM Watson Tone Analyzer, the data has been grouped by user's comments and server status (Failure, Idle) to identify social emotion tone for each user. Table 1 shows a sample of data used in this analysis, with each row representing a unique user, and each column represents the Big Five traits, social emotion tones and server status.

\begin{tabular}{|c|c|c|c|c|c|c|c|c|c|c|}
\hline Openness & Conscientiousness & Extraversion & Agreeableness & Neuroticism & anger & disgust & fear & joy & sadness & Server Status \\
\hline 0.528 & 0.523 & 0.537 & 0.653 & 0.511 & 0.217821 & 0.793375 & 0.501131 & 0.031477 & 70.284936 & Failure \\
\hline 0.252 & 0.063 & 0.037 & 0.266 & 0.989 & 0.542857 & 0.084615 & 0.178302 & 0.224453 & 30.264283 & Failure \\
\hline 0.817 & 0.571 & 0.157 & 0.012 & 0.401 & 0.162798 & 0.166694 & 0.213870 & 0.410916 & 60.220049 & Failure \\
\hline 0.197 & 0.130 & 0.180 & 0.419 & 0.990 & 0.468938 & 0.259794 & 0.350803 & 0.037265 & 50.636412 & Failure \\
\hline 0.155 & 0.079 & 0.081 & 0.226 & 0.975 & 0.539162 & 0.219993 & 0.431932 & 0.011625 & 50.642158 & Failure \\
\hline 0.158 & 0.281 & 0.332 & 0.510 & 0.869 & 0.419015 & 0.162022 & 0.213941 & 0.066892 & 20.686369 & Failure \\
\hline 0.817 & 0.571 & 0.157 & 0.012 & 0.401 & 0.041602 & 0.026298 & 0.141606 & 0.651962 & 20.106500 & Failure \\
\hline 0.058 & 0.038 & 0.147 & 0.375 & 0.989 & 0.449222 & 0.057946 & 0.181654 & 0.158412 & 20.547968 & Idle \\
\hline 0.178 & 0.138 & 0.800 & 0.564 & 0.828 & 0.207497 & 0.096643 & 0.093218 & 0.769316 & 60.162241 & Idle \\
\hline 0.105 & 0.463 & 0.792 & 0.704 & 0.041 & 0.134487 & 0.257145 & 0.195858 & 0.181699 & 90.509379 & Idle \\
\hline 0.589 & 0.479 & 0.147 & 0.339 & 0.828 & 0.360527 & 0.240875 & 0.321188 & 0.117492 & 20.212762 & Idle \\
\hline 0.338 & 0.235 & 0.104 & 0.304 & 0.869 & 0.164107 & 0.015058 & 0.230148 & 0.629562 & 20.356028 & Idle \\
\hline 0.204 & 0.203 & 0.480 & 0.329 & 0.892 & 0.625891 & 0.193692 & 0.242459 & 0.153679 & 90.166561 & Idle \\
\hline 0.689 & 0.968 & 0.805 & 0.465 & 0.029 & 0.246246 & 0.080353 & 0.123761 & 0.807537 & 70.135646 & Idle \\
\hline 0.093 & 0.175 & 0.642 & 0.563 & 0.875 & 0.279503 & 0.045658 & 0.207278 & 0.088724 & 40.505607 & Idle \\
\hline 0.277 & 0.296 & 0.276 & 0.332 & 0.892 & 0.499199 & 0.143897 & 0.269725 & 0.1886664 & 40.285462 & Idle \\
\hline 0.055 & 0.095 & 0.783 & 0.699 & 0.935 & 0.450997 & 0.153940 & 0.263070 & 0.350778 & 80.116282 & Idle \\
\hline
\end{tabular}

Table 1 Example data snapshot used in the analysis 


\subsection{Statistical Analysis}

As part of modelling the users' responses and behaviour, one of the approaches to building a conceptual framework model is to apply linear regression to investigate the relationship between the Big Five personality dimensions and the emotion tones features.

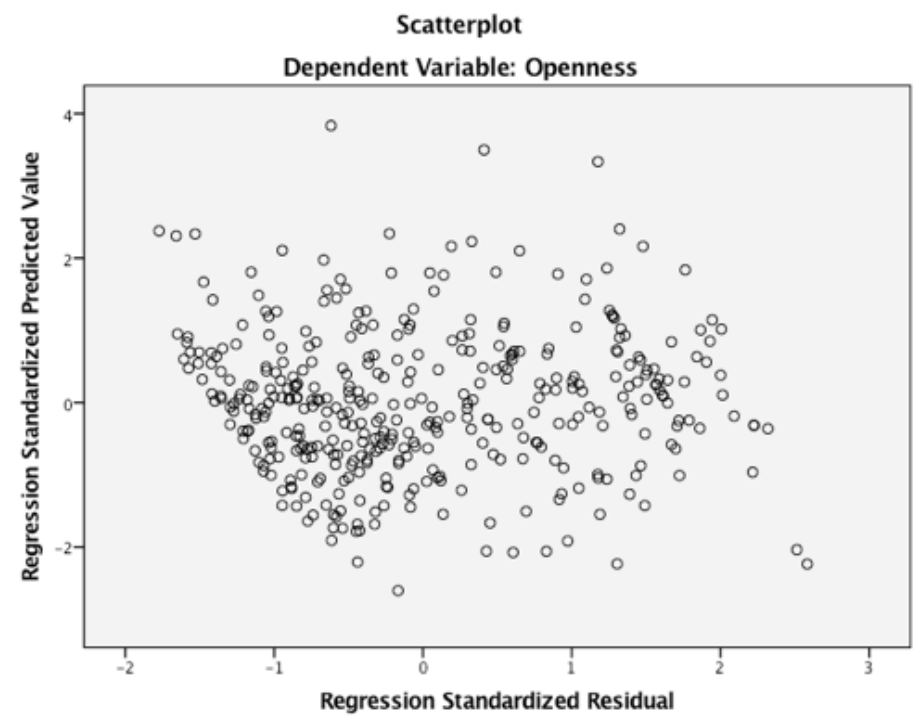

Fig. 3 Scatterplots of Big Five dimension "Openness" (dependent variable) and social emotion tones (independent variables) 
Incorporating Emotion and Personality-Based Analysis in User-Centered Modelling

\section{Scatterplot}

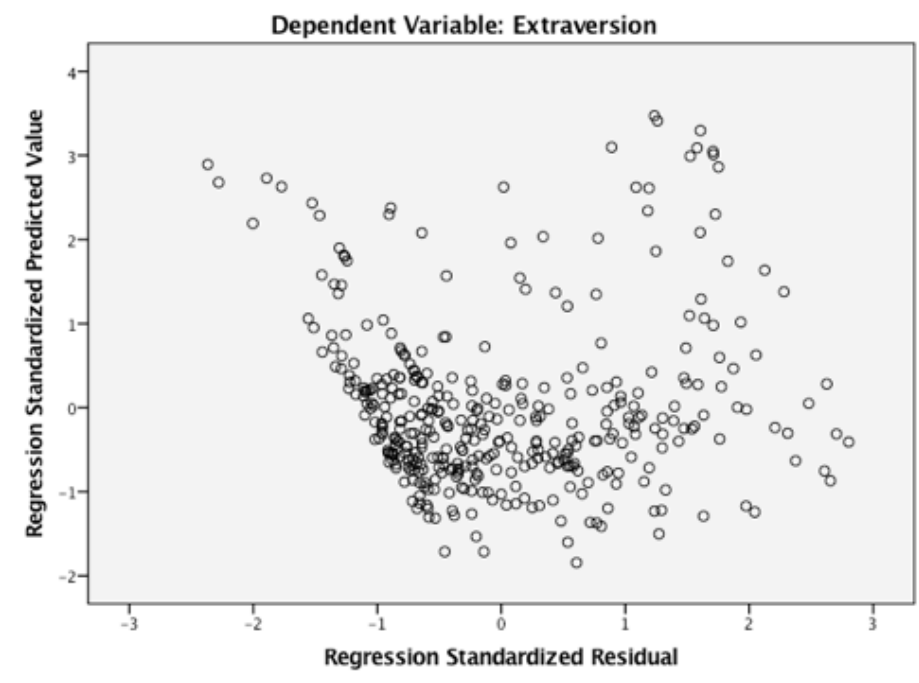

Fig. 4 Scatterplots of Big Five dimension "Extraversion" (dependent variable) and social emotion tones (independent variables)

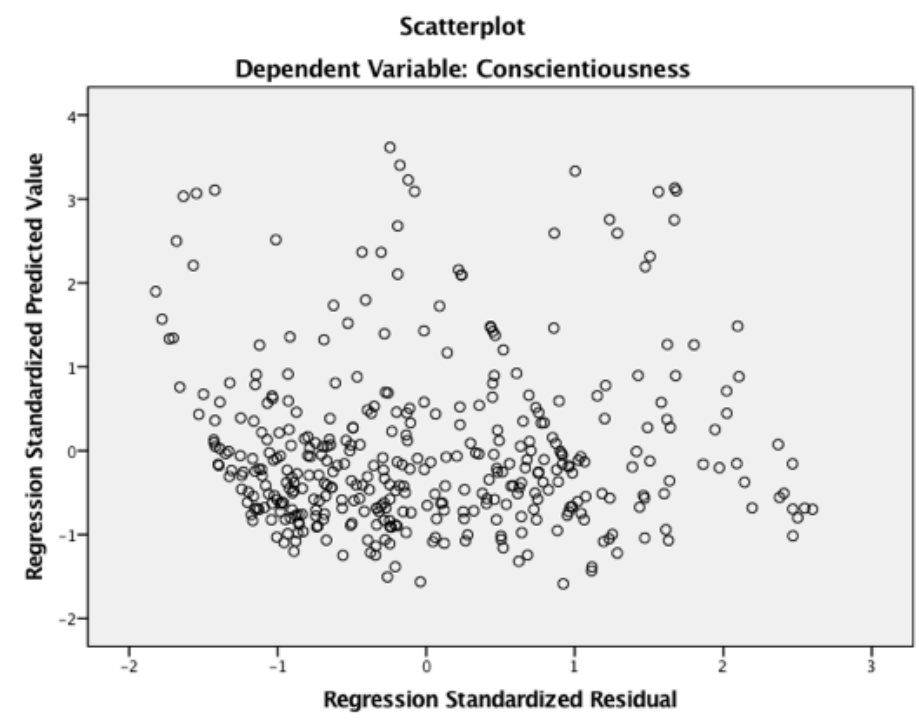

Fig. 5 Scatterplots of Big Five dimension "Conscientiousness" (dependent variable) and social emotion tones (independent variables) 
Mohamed Mostafa, Tom Crick, Ana C. Calderon, and Giles Oatley

Scatterplot

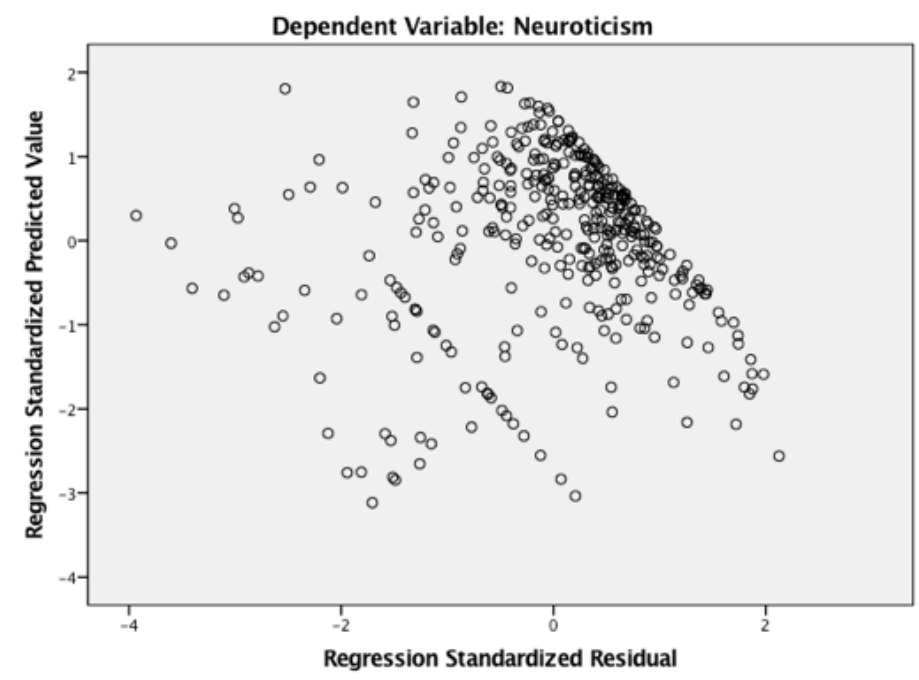

Fig. 6 Scatterplots of Big Five dimension "Neuroticism" (dependent variable) and social emotion tones (independent variables)

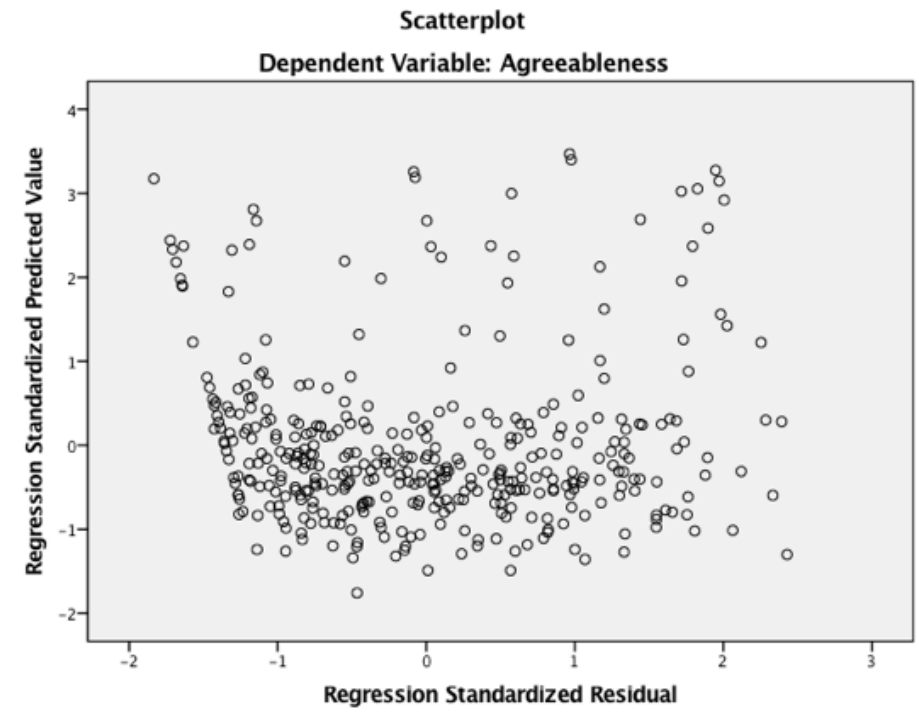

Fig. 7 Scatterplots of Big Five dimension "Agreeableness" (dependent variable) and social emotion tones (independent variables) 
Incorporating Emotion and Personality-Based Analysis in User-Centered Modelling

\begin{tabular}{llllllllllllllll|l}
\hline & \multicolumn{3}{c}{ Openness } & \multicolumn{3}{c}{ Extraversion } & \multicolumn{2}{c}{ Conscientiousness } & \multicolumn{2}{c}{ Agreeableness } & \multicolumn{3}{c}{ Neuroticism } \\
\hline & $\mathrm{B}$ & $\mathrm{t}$ & $\mathrm{Sig}$ & $\mathrm{B}$ & $\mathrm{t}$ & $\mathrm{Sig}$ & $\mathrm{B}$ & $\mathrm{t}$ & $\mathrm{Sig}$ & $\mathrm{B}$ & $\mathrm{t}$ & $\mathrm{Sig}$ & $\mathrm{B}$ & $\mathrm{t}$ & $\mathrm{S}$ \\
(constant) & 0.356 & 3.282 & 0.001 & 0.162 & 1.642 & 0.101 & 0.16 & 1.623 & 0.105 & 0.297 & 2.831 & 0.005 & 0.828 & 9.934 & 0 \\
anger & -0.063 & -0.735 & 0.463 & 0.064 & 0.831 & 0.406 & 0.124 & 1.592 & 0.112 & 0.024 & 0.293 & 0.769 & 0.116 & 1.767 & 0.078 \\
disgust & 0.478 & 4.354 & 0 & 0.114 & 1.142 & 0.253 & 0.255 & 2.551 & 0.011 & -0.061 & -0.574 & 0.566 & -0.363 & -4.303 & 0 \\
fear & 0.065 & 0.534 & 0.594 & 0.172 & 1.549 & 0.122 & 0.04 & 0.356 & 0.722 & 0.093 & 0.783 & 0.434 & -0.023 & -0.241 & 0.81 \\
joy & 0.066 & 0.561 & 0.575 & 0.446 & 4.179 & 0 & 0.436 & 4.058 & 0 & 0.188 & 1.652 & 0.099 & -0.487 & -5.39 & 0 \\
sadness & -0.226 & -2.118 & 0.035 & -0.185 & -1.906 & 0.057 & -0.03 & -0.313 & 0.754 & 0.014 & 0.132 & 0.895 & 0.233 & 2.841 & 0.005 \\
\hline
\end{tabular}

Table 2 Linear regression coefficients

During the analysis, the linear regressions (presented in Table 2 and Figures 3, 4, 5, 6 and 7) do not show significant correlations between the Big Five dimensions and the social emotion tones; however, certain correlations can be highlighted and used as key elements for the model at this stage. The correlation of Openness and Disgust, is 0.479; the correlation of Extraversion and Joy is 0.446 with p-value of zero. Conscientiousness and Joy with 0.436 correlation and Disgust with 0.255. Agreeableness, does not appear to have a high impact in the social emotion parameters, with the highest correlation being 0.188 with Joy, which can be overlooked as a useful factor in the model. Neuroticism and Disgust is -0.363 , Joy is -0.487 and pvalue is zero is both cases; and Sadness with 0.233. All correlation values are $<0.5$; however, it is noticed that Agreeableness does not have a linear relationship with any of the social emotion tones. Furthermore, the social emotion tones that have a potential linear relationship are Disgust, Joy and Sadness, since the three tones have a correlation between $>0.3$ and $<0.5$.

Previous linear regression analysis suggested that the following Big Five dimensions (Openness, Extraversion, Conscientiousness and Neuroticism) have the highest correlation with the social emotion tones (Joy, Sadness and Disgust). For further analysis, the Pearson correlation for the same dataset has been performed to compare the output with the linear regression correlations. As you can see in Table 3, there is no significant correlation in both; however, in the Pearson correlation, Neuroticism has the highest correlation values across emotion tones, especially Anger, Joy and Sadness. Joy does have a correlation with all Big Five dimensions except for Agreeableness which agrees with the previous analysis. However, Disgust does not have a strong correlation with any of the Big Five dimensions, which deviates from the previous analysis.

\begin{tabular}{|c|c|c|c|c|c|}
\hline & Anger & Disgust & Fear & Joy & Sadness \\
\hline & & 0.231 & 043 & 035 & 0.151 \\
\hline onccien & -0 . & -0 & -0 . & 0.267 & 19 \\
\hline & & -0 & & 0.349 & -0.291 \\
\hline & -0.068 & -0.089 & -0.027 & 0.14 & -0.069 \\
\hline Neuroticism & 0.375 & -0.037 & 0.153 & -0.488 & 0.379 \\
\hline
\end{tabular}

Table 3 Pearson correlations 


\section{Key Elements of the Model}

According to the output of the statistical analysis presented in Table 2 (linear regression) and Table 3 (Pearson correlation), the Big Five dimension identified as the key elements from the personality traits are: Openness, Extraversion, Conscientiousness and Neuroticism. The statistical analysis agrees that Agreeableness does not have a significant correlation across any of the social emotion tones. The social emotion tones to be used as key input elements for the proposed model are Joy, Sadness, Anger and Disgust; although the Anger tone did not show any significant correlation in linear regression analysis, the value of the Pearson correlation coefficient is between 0.3 and 0.5 which can be used as input for the model.

$\begin{array}{lr}\text { Correctly classified instances: } & 43(75.44 \%) \\ \text { Incorrectly classified instances: } & 14(24.56 \%) \\ \text { Kappa statistic: } & 0.5295 \\ \text { Mean absolute error: } & 0.3432 \\ \text { Root mean squared error: } & 0.4246 \\ \text { Total number of instances: } & 57\end{array}$

Table 4 Re-evaluation output of proposed model

The dataset used to build this model is based upon a number of users $(n=391)$, eight inputs (Openness, Extraversion, Conscientiousness, Neuroticism, Joy, Sadness, Anger and Disgust) and the class/output variable as the server status (where No: System Failure and Yes: System Idle). As shown in Table 4, the total number of the instances for the testing set is 57 . The output of the model shows a $75.44 \%$ corrected predicted instances and $24.56 \%$ incorrectly classified instances. As this has been performed on a small subset of the overall larger project dataset, the output data is encouraging and provides the infrastructure for further analysis and research to exploit the full dataset.

\section{Conclusions}

This paper presents preliminary results from a larger ongoing theme of research to profile online/digital behaviour [14, 17, 18], which could provide the conceptual framework to improve user experience and computer system architecture design. Social media is now not only being used as a content and sharing platform, but also as a platform for technical support for various of online applications and services. We have produced a model that can predict server status based on personality traits and social emotion tones, by investigating the linear regression and Pearson correlation to identify the key elements to be used as input for the neural network to build this model (Openness, Extraversion, Conscientiousness, Neuroticism, Joy, Sadness, 
Incorporating Emotion and Personality-Based Analysis in User-Centered Modelling

Anger and Disgust). The model developed shows a good potential starting point for further data analysis, with $75 \%$ accuracy in predication based on 57 test cases.

\section{Future Work}

This paper demonstrates the analysis of one such online application that has used Facebook as technical support platform for the users. Such social networks provide substantial textual and interaction datasets for analysis, providing further insight into personality traits and social emotion tones. We are also interested in profiling complex behaviours and psychopathies using social network analysis, particularly for crime informatics $[15,16]$. Previous work in this space analysed the document uploading behaviour (such as motivation letters, and social media interactions) of the applicants of the international scholarship mobility portal; by examining the upload footprint for the users we were able to determine several classes of behaviour [19].

The outcome of the model produced during this work provides the following recommendations for future research to further incorporate emotion and personalitybased analysis in user-centered modelling, as well as building upon the availability of high-quality, low-cost and adaptable tools provided by the IBM Watson Developer Cloud (e.g. IBM Watson Tone Analyzer and IBM Watson Personality Insights tools), provide significant further opportunities to integrate linguistic analysis into this research domain:

- Expanding the dataset by gathering more data from similar types of interactions, as well as technical queries;

- Annotate and categorise the dataset by gender to investigate the relationship between gender and emotion raised by the user in different computer system statuses;

- Explore different computer events not only limited to Idle and Failure, but including more complex events e.g. account hacked, system speed, unexpected error and unsaved data.

\section{References}

[1] J. Biel and D. Gatica-Perez. The YouTube Lens: Crowdsourced Personality Impressions and Audiovisual Analysis of Vlogs. IEEE Transactions on Multimedia, 15(1):41-55, 2012.

[2] B. Blamey, T. Crick, and G. Oatley. R U :-) or :-( ? Character- vs. Word-Gram Feature Selection for Sentiment Classification of OSN Corpora. In Research and Development in Intelligent Systems XXIX, pages 207-212. Springer, 2012.

[3] B. Blamey, T. Crick, and G. Oatley. 'The First Day of Summer': Parsing Temporal Expressions with Distributed Semantics. In Research and Development in Intelligent Systems XXX, pages 389-402. Springer, 2013. 
[4] J. Block. The Five-Factor Framing of Personality and Beyond: Some Ruminations. Psychological Inquiry, 21(1):2-25, 2010.

[5] P. T. Costa and R. R. McCrae. Revised NEO Personality Inventory (NEO-PI-R) and NEO Five-Factor Inventory (NEO-FFI) Manual. Psychological Assessment Resources, 1992.

[6] David Lazer et al. Computational Social Science. Science, 323(5915):721723, 2009.

[7] H. J. Eysenck. Four ways five factors are not basic. Personality \& Individual Differences, 13(6):667-673, 1992.

[8] L. A. Fast and D. C. Funder. Personality as Manifest in Word Use: Correlations with Self-Report, Acquaintance Report, and Behavior. Journal of Personality \& Social Psychology, 94(2):334-346, 2008.

[9] C. Fellbaum. Encyclopedia of Language \& Linguistics, chapter WordNet(s), pages 665-670. Elsevier, 2nd edition, 2006.

[10] F. Iacobelli, A. Gill, S. Nowson, and J. Oberlander. Large Scale Personality Classification of Bloggers. In Proceedings of the 4th International Conference on Affective Computing and Intelligent Interaction (ACII'11), pages 568-577, 2011.

[11] R. Lambiotte and M. Kosinski. Tracking the Digital Footprints of Personality. Proceedings of the IEEE, 102(12):1934-1939, 2014.

[12] F. Mairesse, M. Walker, M. Mehi, and R. Moore. Using Linguistic Cues for the Automatic Recognition of Personality in Conversation and Text. Journal of Artificial Intelligence Research, 30:457-500, 2007.

[13] W. T. Norman. Toward an adequate taxonomy of personality attributes: Replicated factor structure in peer nomination personality ratings. Journal of Abnormal \& Social Psychology, 66(6):574-583, 1963.

[14] G. Oatley and T. Crick. Changing Faces: Identifying Complex Behavioural Profiles. In Human Aspects of Information Security, Privacy and Trust, volume 8533 of LNCS, pages 282-293. Springer, 2014.

[15] G. Oatley and T. Crick. Exploring UK Crime Networks. In Proceedings of 2014 International Symposium on Foundations of Open Source Intelligence and Security Informatics (FOSINT-SI 2014). IEEE Press, 2014.

[16] G. Oatley and T. Crick. Measuring UK Crime Gangs: A Social Network Problem. Social Network Analysis and Mining, 5(1):33, 2015.

[17] G. Oatley, T. Crick, and D. Bolt. CCTV as a Smart Sensor Network. In Proceedings of 13th IEEE International Conference on Dependable, Autonomic and Secure Computing (DASC-2015). IEEE, 2015.

[18] G. Oatley, T. Crick, and R. Howell. Data Exploration with GIS Viewsheds and Social Network Analysis. In Proceedings of 23rd GIS Research UK Conference (GISRUK 2015), 2015.

[19] G. Oatley, T. Crick, and M. Mostafa. Digital Footprints: Envisaging and Analysing Online Behaviour. In Proceedings of 2015 Symposium on Social Aspects of Cognition and Computing Symposium (SSAISB 2015), 2015. 
Incorporating Emotion and Personality-Based Analysis in User-Centered Modelling

[20] J. Oberlander and A. J. Gill. Individual differences and implicit language: Personality, parts-of-speech and pervasiveness. In Proceedings of the 26th Annual Conference of the Cognitive Science Society, pages 1035-1040, 2004.

[21] J. Oberlander and A. J. Gill. Language with character: A stratified corpus comparison of individual differences in e-mail communication. Discourse Processes, 42(3):239-270, 2006.

[22] S. V. Paunonen and D. N. Jackson. What is beyond the Big Five? Plenty! Journal of Personality, 68(5):821-836, 2000.

[23] D. Peabody and L. Goldberg. Some determinants of factor structures from personality-trait descriptor. Journal of Personality \& Social Psychology, 57 (3):552-567, 1989.

[24] J. Pennebaker and L. King. Linguistic styles: language use as an individual difference. Journal of Personality \& Social Psychology, 77(6):1296-1312, 1999.

[25] J. W. Pennebaker, M. E. Francis, and R. J. Booth. Linguistic Inquiry and Word Count. Erlbaum Publishers, 2001.

[26] K. R. Scherer. Emotion as a Multicomponent Process: A model and some cross-cultural data. Review of Personality \& Social Psychology, 5:37-63, 1984.

[27] S. Schiaffino and A. Amandi. Intelligent User Profiling. In Artificial Intelligence: An International Perspective, volume 5640 of LNCS, pages 193-216, 2009.

[28] Y. R. Tausczik and J. W. Pennebaker. The Psychological Meaning of Words: LIWC and Computerized Text Analysis Methods. Journal of Language \& Social Psychology, 29(1):24-54, 2010. 